in the data were detected by quantitative data mining algorithms, the reporting odds ratio.

Results A total of 1,763 reports related to efavirenz were analysed. The average age were 46.42 years. Two cases were fatal outcome. Significant signals of the adverse events were detected with hepatitis, exfoliative dermatitis and Stevens Johnson syndrome. Drug eruption was also detected.

Conclusions Serious reactions from efavirenz were hepatitis, exfoliative dermatitis and Stevens Johnson syndrome. They made fatal and life threatening outcome. The recommendation is further specific monitoring to reduce serious risk in efavirenz use.

Disclosure of interest statement Serious reactions from efavirenz were hepatitis, exfoliative dermatitis and Stevens Johnson syndrome. They made fatal and life threatening outcome. The recommendation is further specific monitoring to reduce serious risk in efavirenz use.

\section{P17.34 SURVIVAL TREND AND IMPACT OF ADVERSE DRUG REACTIONS DURING HAART ON SURVIVAL FUNCTION IN HIVIAIDS PATIENTS}

${ }^{1}$ KashifUllah Khan*, 'Amer Hayat Khan, ${ }^{1}$ Syed Azhar Syed Sulaiman, ${ }^{2}$ Chow Ting Soo. ${ }^{1}$ School of Pharmaceutical Sciences, Universiti Sains Malaysia, Penang, Malaysia; ${ }^{2}$ Infectious Disease Department, General Hospital Pulau Pinang, Penang, Malaysia

\subsection{6/sextrans-2015-052270.612}

Aims and objective Current study is aimed to observe survival trend in HIV/AIDS patients and to explore the impact of ADRs experienced during HAART on survival trends of the patients.

Method An observational retrospective study of all patients diagnosed of HIV infection and on HAART therapy from Jan 2007 to Dec 2012 was conducted at infectious disease department of Hospital Pulau Pinang, Malaysia. Patient socio-demographic details along with clinical features were recorded. The survival function was observed on Kaplan-Meir survival analysis and Cox-regression for survival function. Data was descriptively analysed by using statistical package for social sciences (SPSS 20).

Results Out of 792 patients that underwent HAART therapy, $607(76.6 \%)$ were male and $185(23.3 \%)$ were female patients. The probability of 6 years survival was compared where the overall median follow up time of all patients was 36 months or 3 years (inter-quartile range 33.5-38.4). On Kaplan-Meir survival function analysis, the survival probability among female patients (52\%) was higher than males $(48 \%)$ until 48 months after which the male patients showed better survival ( $p$ 0.194). Better survival probability were observed among non-smokers ( $p$ 0.194), non-alcoholics (p 0.002), and non-drug abusers ( $\mathrm{p}<$ 0.001). Overall $338(42.6 \%)$ patients had experienced adverse drug reactions where a total number of 449 (56.7\%) adverse drug reactions were reported among which 329 (73.2\%) occurred in males and $120(26.8 \%)$ in female patients. The survival probability with significant association $(\mathrm{p}<0.001)$ among patients with absence of ADR were reported higher on KaplanMeir survival. On Cox-regression survival analysis, Alcoholic patients (HR 1.14, p 0.02), drug abusers (HR 1.38, p 0.01) and patients with ADRs (HR 0.65, p < 0.001) shows a higher risk for death with higher Hazard ratio.

Conclusion The study indicates that a patient's life-style and occurrence of ADRs has a direct impact on survival probability in HIV/AIDS patients which shows a greater risk to death and poor survival. However, a multicenter study with a large sample size may provide us with better understanding of this relationship.

Disclosure of interest All authors are aware that current abstract is submitted to World STI and HIV 2015 Congress. The abstract has not been submitted elsewhere. The authors have no conflict of interest.

\section{P17.35 COST-EFFECTIVENESS ANALYSIS OF ABACAVIR-BASED AND TENOFOVIR-BASED REGIMENS IN SINGAPORE}

YY Ong, G Hoo*, HL Law, OT Ng. Tan Tock Seng Hospital, Singapore

\subsection{6/sextrans-2015-052270.613}

Introduction Human immunodeficiency virus (HIV) infection has evolved into a chronic disease with the use of highly active antiretroviral therapy (HAART). As survival rates improve and the duration of treatment increases, cost of treatment will be a major factor affecting the choice of HAART.

Objective To assess the cost-effectiveness of abacavir-based and tenofovir-based regimens in Singapore.

Methods This was a single-centre, retrospective study. Data was obtained from online medical records and hardcopy case files. HIV-positive patients on each regimen were matched according to age group, gender, race, body mass index and HAART. An incremental cost-effectiveness ratio (ICER) analysis was performed to evaluate the cost-effectiveness between abacavir (ABC)-based and tenofovir (TDF)-based regimens between the evaluation period of week 12 to 36 and week 37 to week 60 . Costs were reported in Singapore dollars (S\$).

Results Two hundred and thirty patients were analysed $(82.2 \%$ Chinese, $91.3 \%$ male, age: $46.0 \pm 13.0$ years old), 115 patients in each group. The most common used combinations were $\mathrm{ABC}$, lamivudine (3TC) and efavirenz (EFV) (76.5\%), and TDF, 3TC and EFV (78.2\%); followed by ABC, 3TC, boosted atazanavir (ATV/r) (13.0\%), and TDF, 3TC and ATV/r (11.3\%). Majority of the patients in each arm were at least $95 \%$ adherent to their medication regimens $(93.0 \%$ and $91.3 \%$ for $\mathrm{ABC}$ and $\mathrm{TDF}$ group respectively). For both evaluation periods, more patients in $\mathrm{ABC}$ group obtained undetectable viral load $(77.4 \%$ vs $59.1 \%$ and $81.7 \%$ vs $76.5 \%)$. The ICER value was $-S \$ 13348.72$ for the period of week 12 to 36 and $-\$ \$ 19085.37$ for the period of week 37 to week 60 .

Conclusion ABC-based regimen was found to be more costeffective than TDF-based regimen with similar virologic control rates in HIV patients in Singapore. This can translate to public healthcare cost savings in the near future.

\section{P17.36 EFFECTIVENESS, SAFETY AND TOLAREBILITY PROFILE OF STRIBILD ${ }^{\circledR}$ (ELVITEGRAVIR/COBICISTAT/ EMITRICITABINE/TENOFOVIR DISOPROXIL FUMARATE) IN HIV-1-INFECTED PATIENTS IN THE CLINICAL SETTING}

J Heiko*, T Laura, R Slobodan. Praxis Jessen²+Kollegen, Berlin, Germany

\subsection{6/sextrans-2015-052270.614}

Introduction The efficacy of Stribild ${ }^{\circledR}$, an integrase strand transfer inhibitor (INSTI) -based STR, has been evaluated in randomised clinical trials. However, restricted selection criteria, monitoring frequency and selection bias hamper data extrapolation in routine practice. Here we analysed the virologic 
outcomes, safety and tolerability profile of Stribild ${ }^{\circledR}$ in clinical practice.

Methods Retrospective monocentric analysis on HIV-1-infected patients, who started with or were switched to Stribild ${ }^{\circledR}$. The percentage of patients remaining free of therapeutic failure was estimated using the time-to-loss-of-virologic-response (TLOVR) algorithm, by intent-to-treat analysis.

Results We analysed the data of 197 patients (56 ART-naïve and 141 treatment-experienced patients). At the end of follow-up (median 33 months), $87.3 \%$ of treatment-naïve and $80.3 \%$ of treatment-experienced patients remained free of therapeutic failure. A total of 17 patients stopped treatment with Stribild ${ }^{\circledR}$, $5.4 \%(3 / 56)$ of them were treatment-naïve and 9.9\% (14/141) were treatment-experienced patients. The Stribild ${ }^{\circledR}$ therapy was discontinued in 2 because of VF, loss to follow-up in 4, and drug-drug interactions in 2 patients. Adverse events were in 7 $(3.6 \%)$ patients the reason to switch from therapy with Stribild ${ }^{\circledR}$ and further $2(1.0 \%)$ patients decided personally to switch. In two patients novel resistances in integrase-gene $(\mathrm{N} 155 \mathrm{H}$ and S119R) emerged. In one further patient with VF two novel mutations in the RT-gene were observed when compared to historical genotypic test result (V106I/M and M184V).

Conclusion In treatment-naïve patients effectiveness of Stribild ${ }^{\circledR}$ was consistent with data obtained in clinical trials. The safety and tolerability profile as well as resistance development confirmed clinical efficacy of Stribild ${ }^{\circledR}$ in a daily practice setting.

Disclosure of interest statement Heiko Jessen received honoraria related to speakers' activities and consulting from ViiV Healthcare, AbbVie Germany, Bristol-Myers Squibb and Gilead Sciences, grants from MSD Sharp and Dohme and ViiV Healthcare and an additional grant from Gilead Sciences for this study.

\section{P17.37 SAFETY AND EFFICACY OF ELVITEGRAVIR/COBICISTAT/ EMTRICITABINE/TENOFOVIR DISOPROXIL FUMARATE IN TREATMENT-NAÏVE JAPANESE PATIENTS WITH HIV-1 INFECTION}

K Yajima*, H Yagura, S Yukawa, K Hirota, M Ikuma, D Kasai, D Watanabe, Y Nishida, T Uehira, T Shirasaka. AIDS Medical Center, National Hospital Organization Osaka National Hospital

\subsection{6/sextrans-2015-052270.615}

Introduction Elvitegravir/Cobicistat/Emtricitabine/Tenofovir Disoproxil Fumarate $(\mathrm{E} / \mathrm{C} / \mathrm{F} / \mathrm{TDF})$, a single tablet regimen, is expected as a pillar of antiretroviral therapy. Although, there have been few reports on antiviral effects and adverse events in cases of its initial therapy among Asian patients.

Methods We retrospectively reviewed medical records of patients treated with a single tablet regimen $\mathrm{E} / \mathrm{C} / \mathrm{F} / \mathrm{TDF}$ once daily between May 2013 and October 2014. Patients with insufficient data and those with conditions such as chronic renal failure or viral hepatitis were excluded.

Results In the 106 subjects who received E/C/F/TDF: 99\% men, $31 \%$ viral load $(\mathrm{VL}) \geqq 100,000$ copies $/ \mathrm{mL}$ (c/mL). Median characteristics were: age $37 \mathrm{yrs}$, VL $4.85 \log _{10} \mathrm{c} / \mathrm{mL}$, CD4 count 260 cells $/ \mu \mathrm{L}$. Mean change in CD4 count at Week 48 was +247 cells $/ \mu \mathrm{L}, \mathrm{VL}-2.7 \log _{10} \mathrm{c} / \mathrm{mL}$. The serum creatinine level increased by $0.09 \mathrm{mg} / \mathrm{dL}$ : however, no further increase was seen during the observation period. There was no report of proximal renal tubulopathy including Fanconi Syndrome. Liver function tests and lipid markers demonstrated no significant changes. A total of 21 adverse events (AEs) were observed in 18 subjects (17.0\%). The most common AEs were neurologic symptoms (dizziness, headaches, vivid dreams etc.), which occurred in 12 subjects: although, the symptoms either disappeared or ameliorated within 4 weeks in all subjects. No single AE led to discontinuation of more than 1 subject showed renal dysfunction. Virologic failure with resistance occurred in $3.8 \%$.

Conclusion High virologic response was seen in patients receiving $\mathrm{E} / \mathrm{C} / \mathrm{F} / \mathrm{TDF}$. This regimen was well tolerated, and no unique AEs were occurred, compared to the previous reports. These data support the use of $\mathrm{E} / \mathrm{C} / \mathrm{F} / \mathrm{TDF}$ as a potential new regimen for initial treatment of Japanese patients with HIV-1 infection. Disclosure of interest statement Authors do not have any commercial or other association that might pose a conflict of interest.

\section{P17.38 PLASMA DRUG LEVELS OF NEVIRAPINE PREDICT VIROLOGICAL RESPONSE IN PATIENTS RECEIVING TREATMENT IN KENYAN HOSPITALS}

1,2 M Kimulwo*, 1,2RC Kitawi, 1,2RS Mwatelah, 1,3 R Aman, 1,3 G Masankwa, 1,4 B Ogutu, ${ }^{1} \mathrm{~J}$ Okendo, ${ }^{4} \mathrm{RM}$ Lwembe, ${ }^{2} \mathrm{M}$ Muigai, ${ }^{1,4} \mathrm{~W}$ Ochieng. ${ }^{1}$ Center for Research in Therapeutic Sciences, Strathmore University, Nairobi, Kenya; ${ }^{2}$ Institute of Tropical Medicine and Infectious Diseases, JKUAT, Kenya; ${ }^{3}$ African Centre for Clinical Trials, Nairobi, Kenya; ${ }^{4}$ Kenya Medical Research Institute, Nairobi, Kenya

\subsection{6/sextrans-2015-052270.616}

Introduction Treatment failure is a key challenge in the management of HIV-1 infection. We examined the association of nevirapine blood levels with virologic treatment outcome and adherence among Kenyan patients on antiretroviral therapy (ART).

Methods A cross-sectional study involving 58 subjects receiving nevirapine as part of ART regimen. Clinical, demographic and adherence data were captured using structured questionnaires. Five millilitres of EDTA blood was collected at 1, 4 and $24 \mathrm{~h}$ post-dosing and used for quantification of nevirapine levels using High-Performance Liquid Chromatography (HPLC). Plasma viral load was determined on $m 2000$ Abbott RealTime HIV-1 assay platform and used to determine virologic treatment failure (VF). Results Median duration of ART was 42 months and $43.1 \%$ of the patients had VF with a mean viral load of $4.24 \log _{10}$ copies. Measured at $1 \mathrm{hr}(\log 2.93, \mathrm{p}=0.003)$ and at $4 \mathrm{hrs}(\log 3.07$, $\mathrm{p}<0.001)$ post dosing, Nevirapine levels were significantly lower for VF than non-VF patients and were significantly associated with virologic response $\left(c^{2} \mathrm{p}<0.001\right)$. These nevirapine levels at $1 \mathrm{hr}\left(\mathrm{R}^{2}=0.218, \mathrm{p}=0.002\right)$ and at $4 \mathrm{hrs}\left(\mathrm{R}^{2}=\right.$ $0.156, \mathrm{p}=0.001$ ) were significantly and inversely correlated with same day VL in a Spearman's rho model. Up to $53.4 \%$, $24.1 \%$ and $22.4 \%$ of the patients had good, fair and poor adherence respectively, with adherence being significantly associated with plasma nevirapine levels at $1 \mathrm{hr}\left(\mathrm{c}^{2} \mathrm{p}=0.001\right)$ and at $4 \mathrm{hr}$ $\left(c^{2} p=0.021\right)$. No significant associations were found at $24 \mathrm{hrs}$ post nevirapine dosing.

Conclusion Majority of VF patients attained nevirapine levels in plasma that were significantly lower than non-VF patients. These patients were also more likely to have poor adherence than virologic responders with higher nevirapine levels. Suboptimal exposures to nevirapine may be mitigated partly by improving adherence support mechanisms. Additional investigations should focus on pharmacogenetics and other factors influencing optimal drug uptake in blood.

Disclosure of interest statement ISSTDR and IUSTI recognise the considerable contribution that industry partners make to professional and research activities. We also recognise the need for 